\title{
A HYPERSPECTRAL BASED METHOD TO DETECT CANNABIS PLANTATION IN INACCESSIBLE AREAS
}

\author{
M. Houmi, B. Mohamadi*, T. Balz
}

State Key Laboratory of Information Engineering in Surveying, Mapping and Remote Sensing, Wuhan University, People's Republic of China, houmi2mohamed@gmail.com, (bh.mo, balz)@whu.edu.cn.

KEY WORDS: EO-1 Hyperion, Hyperspectral data, preprocessing, Cannabis, Morocco, Spectral Angle Mapper (SAM).

\begin{abstract}
:
The increase in drug use worldwide has led to sophisticated illegal planting methods. Most countries depend on helicopters, and local knowledge to identify such illegal plantations. However, remote sensing techniques can provide special advantages for monitoring the extent of illegal drug production. This paper sought to assess the ability of the Satellite remote sensing to detect Cannabis plantations. This was achieved in two stages: 1- Preprocessing of Hyperspectral data EO-1, and testing the capability to collect the spectral signature of Cannabis in different sites of the study area (Morocco) from well-known Cannabis plantation fields. 2- Applying the method of Spectral Angle Mapper (SAM) based on a specific angle threshold on Hyperion data EO-1 in well-known Cannabis plantation sites, and other sites with negative Cannabis plantation in another study area (Algeria), to avoid any false Cannabis detection using these spectra. This study emphasizes the benefits of using hyperspectral remote sensing data as an effective detection tool for illegal Cannabis plantation in inaccessible areas based on SAM classification method with a maximum angle (radians) less than 0.03 .
\end{abstract}

\section{INTRODUCTION}

Cannabis is the most highly demanded and widely trafficked illegal drug in the world. Hence, illegal plantation of Cannabis is considered as one of the most challenging problems in many countries in the modern world (CBRC, 2007).

To detect the illegal plantation of Cannabis, on-ground surveys are difficult because of inaccessibility of illegal plantation areas. Most countries depend on aerial surveillance and local knowledge of expected planting areas. However, these methods cover limited areas and are not suitable for spontaneous and new plantation areas. Besides, it is intensively time and money consuming. Therefore, using satellite remote sensing data can be a better solution to overcome these challenges due to its wide coverage, high temporal resolution, and low cost compared to aerial visual inspections (Wang, 2013).

High spatial resolution remote sensing images had been successfully used to detect Cannabis by visual image interpretation methods such as WorldView-2, and GeoEye (UNODC, 2003), and IKONOS, and Quick Bird (CBRC, 2007). As well as to verify Cannabis eradication (UNODC, 2003 and UNODC, 2010). However, the high spatial resolution data is costly, making the full coverage of the crop fields in a large area an expensive exercise. In comparison to high spatial resolution images costs, the hyperspectral data of Earth Observing 1 (EO1) mission, carried by the National Aeronautics and Space Administration (NASA) (EO-1) satellite, is free and can be downloaded from the United States Geological Survey (USGS) website (USGS, 2017).

This advantage gives us the opportunity to test the capability of using hyperspectral data to collect the spectral signature of Cannabis from well-known Cannabis plantation fields and test it to ensure its detection capabilities in unknown illegal Cannabis plantation fields. Although Hyperion data has a medium spatial resolution $(30 \mathrm{~m})$, its high spectral resolution of the $10 \mathrm{~nm}$ spectral interval provides an effective measurement to distinguish Cannabis from other types of vegetation based on its spectra in the visible near-infrared region (VNIR), (Beck, 2003).

Collecting spectra could be done in three ways; by a hand-held spectrometer in the field or the laboratory, or by extracting pure endmembers from remote sensing image pixels. In our case, we selected the third method to extract Cannabis spectra for two reasons: first, there are many difficulties related to collecting Cannabis spectra from the field due to inaccessibility and security issues. Second, we assumed that extracting different spectra from an image after all preprocessing procedures, that has all natural effects including different plant wetness variables, and soil properties could be more efficient rather than collecting spectra in a laboratory.

\section{STUDY AREAS}

Morocco was selected as a study area to collect Cannabis spectra and testing the selected spectra. The selection of Morocco was based on: first, the large extend of Cannabis plantation in the northern region of Morocco, where Cannabis cultivation is legal, as the Cannabis plantation covers more than $20,000 \mathrm{~km}^{2}$ (UNODC, 2003). Second, ground-truth data is available from the survey report of Cannabis cultivation and production in Morocco in 2003 by the UNODC (UNODC, 2003). The selected study area for Cannabis spectra collection is in Chefchaouen Province in the northwest of Morocco. According to the Moroccan Cannabis survey 2003, Chefchaouen is a dominant Cannabis cultivating province in Morocco; Cannabis is cultivating on an area of about 67,000 ha. This province has more than $52 \%$ of the total Cannabis cultivation area of the country in 2003 (UNODC, 2003).

The second study area is Algeria, where Cannabis plantation is illegal. Some parts in the Algerian territory were selected based

\footnotetext{
* Corresponding author
} 
on our personal experience that no illegal Cannabis plantation in those areas. This process aimed to confirm the applicability of our extracted spectra by demonstrating the absence of false Cannabis detection.

\section{METHODOLOGY}

\subsection{Data}

The well-calibrated EO-1 Hyperion images Level L1R data were selected for this study as represented in table 1, two images in Morocco, and one image in Algeria (USGS, 2017). The EO-1 satellite was launched on November 21, 2000, to an orbit at $705 \mathrm{~km}$ altitude. Hyperion data has 242 bands produced by two spectrometers. One operates 70 bands in the VNIR range between $356-1058 \mathrm{~nm}$, and the other operates 172 bands in the SWIR range between $852-2577 \mathrm{~nm}$. The image swath width is about $7.65 \mathrm{~km}$ and has a spatial resolution of $30 \mathrm{~m}$ (Beck, 2003).

\begin{tabular}{|l|c|c|}
\hline Country & Image & Acquisition Date \\
\hline Morocco & EO12010362003131110PZ & June 28, 2003 \\
& EO12000362009190110T4 & July 9, 2009 \\
Algeria & EO11940352004213110PY & June 31, 2004 \\
\hline
\end{tabular}

Table 1. Study EO-1 Hyperion data

\subsection{Images Preprocessing}

Before collecting Cannabis spectra, it is necessary to do some preprocessing steps for Hyperion data to correct it radiometrically and atmospherically. We used ENVI 5.3 software (ENVI Development Team, 2015). To remove noise from different sources to increase the detection capability and ensure the quality of the collected spectra, those steps area representing in figure 1.

The preprocessing steps used in this study are: correct bad pixels, remove bad bands, and vertical stripes, smile effect correction, apply atmospheric correction, and finally apply a geometric correction to Hyperion L1R data.

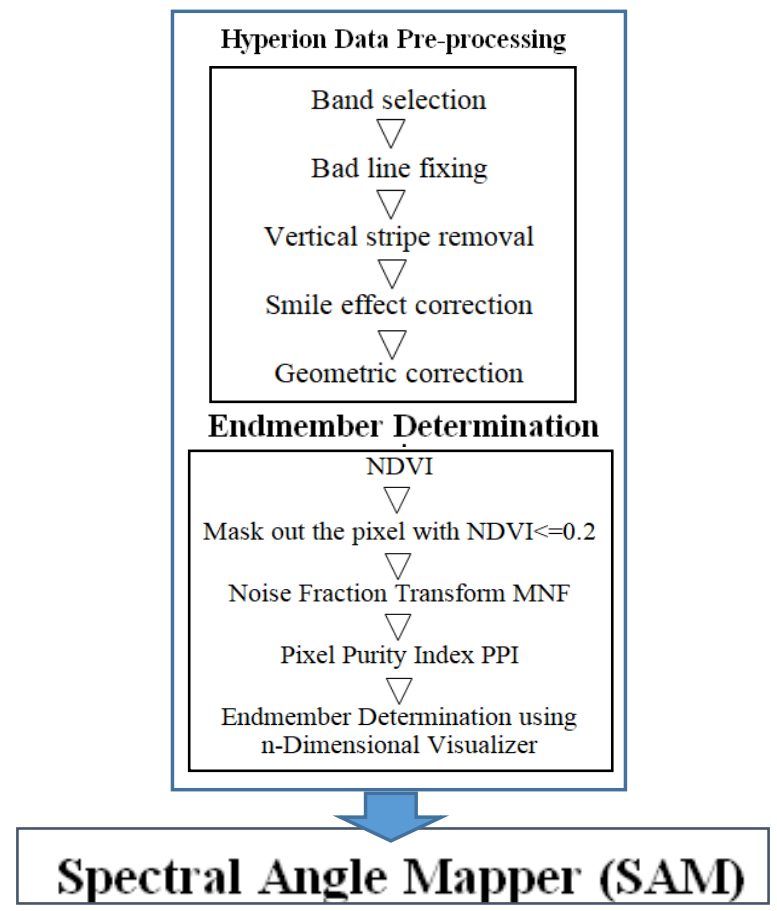

Figure 1. Methodology workflow
3.2.1 Remove Bad Bands: Hyperion data contain 242 spectral bands in total. However, the bands 1 to 7, 58 to 76, and 225 to 242 have no information (Beck, 2003). Hence, they have been excluded from further steps. Also, there are four remaining bands in the overlap between the two spectrometers. These are VNIR bands $56(915.7 \mathrm{~nm})$ and $57(925.9 \mathrm{~nm})$ and SWIR bands $77(912.5 \mathrm{~nm})$ and 78 (922.6 nm), (Tsai, F 2007). Hence, we eliminate the two SWIR bands to obtain 196 unique bands.

Azaria et al., (2012) has stated that the best spectral identification wavelengths for Cannabis plants are $530-550 \mathrm{~nm}$, 670- $680 \mathrm{~nm}$ and 705-720 nm. Hence, we have selected the 50 available bands in VNIR region (bands 8 to 57) to collect spectra and detect Cannabis plantation.

3.2.2 Correct Bad Pixels and Vertical Stripes: For the selected 50 bands of VNIR; bad lines that have lower DN values among the surrounding pixels were corrected by replacing their DN values with the average DN values of their immediate neighboring pixels (Jupp et al., 2003). Then, vertical stripes were removed using the global destriping. Where, the statistical moments of each column are modified to match those, for the whole image in each band.

3.2.3 Remove Smile Effects: The smile effect is an acrosstrack wavelength shift from center wavelength due to the change of dispersion angle with field position (Goodenough et al., 2003). Cross-Track Illumination Correction was used to remove the smile effect from the study images in the ENVI software. The correction of smile effect was then tested by using the Minimum Noise Fraction (MNF) transform (Figure 2), because smile effect could lead to a brightness gradient appearing in the first eigenvalue image after MNF transform, (Somers et al., 2011).

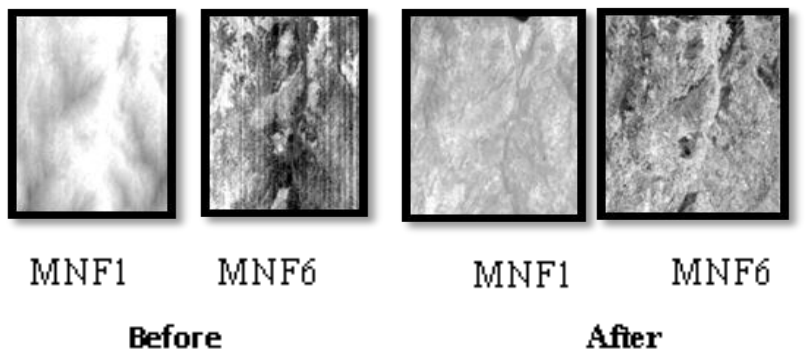

Figure 2. Testing the Effects of destriping using the MNF Transform

3.2.4 Atmospheric Correction: This study used Fast Lineof-sight Atmospheric Analysis of Spectral Hypercubes (FLAASH), in ENVI software to calibrate the at-sensor radiance data to land surface reflectance. As Hyperion level, L1R data are in units of $\mathrm{W} /(\mathrm{m} 2 \mu \mathrm{m} \mathrm{sr}) \times 40$ for the VNIR while the FLAASH atmospheric correction software uses units of $\mu \mathrm{W} /(\mathrm{cm} 2 \mathrm{~nm} \mathrm{sr})$, the scale factor of ten was used when running the FLAASH model (Datt, B, 2003).

3.2.5 Geometric Correction: The Hyperion L1R data which used in this study was geometrically corrected based on Landsat7 images during the same period of Hyperion images acquisition. Images were projected to UTM WGS 29N Zone for Morocco, and Zone $31 \mathrm{~N}$ for Algeria. 


\subsection{Endmember Determination}

Each pixel in an image could contain various endmembers (that called mixed pixel), or contain only one endmember (that called pure pixel), based on different spatial and temporal variability in the condition of scene components and differential illumination conditions (Somers et al., 2011).

To extract Cannabis endmembers in this study, we used a method that is based on supervised endmember-selection SAM. Where, uses an n-D angle to match pixels to reference spectra and compares the angle between the end member spectrum vector and each pixel vector in $n-D$ space (Kale, 2016). We used the distribution maps of Cannabis cultivation in Morocco which has reported in (UNODC, 2003), as ground-truth data for Cannabis spectra collection from a Hyperion image of the same time of the report collected data (figure 3 ).

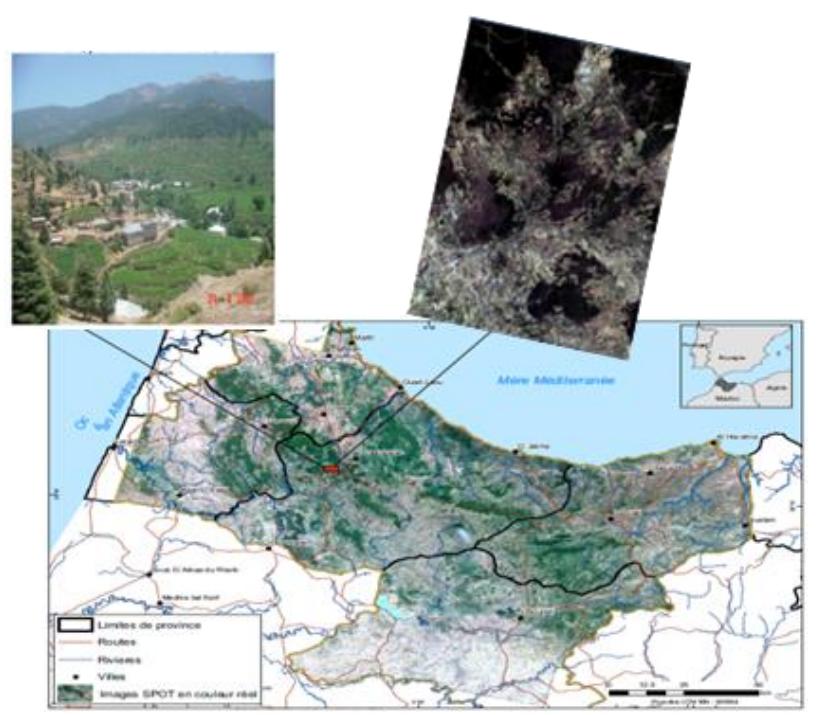

Figure 3. A tested area for Cannabis endmember selection based on ONUDC/ICMP Morocco Cannabis Survey report (UNODC, 2003)

Cannabis distribution maps from the report were geo-referenced and overlaid on geocoded Hyperion image to collect the spectra from obvious Cannabis fields in the report. The process started by calculating the Normalized Difference Vegetation Index (NDVI) using Band 31 (660.85 nm) and Band 44 (793.13 nm). All pixels with NDVI values less than 0.2 were isolated from the image to indicate vegetated Areas only (Tsai et al. 2007; Noujdina and Ustin, 2008). Then, MNF transformation was used to determine the inherent dimensionality of the image data, and to segregate noise in the data only for the first six MNF bands with high eigenvalues (higher spectral information content). Other bands were eliminated due to their low eigenvalues as those bands were carried more noise (Boardman, 1994).

Next, the Pixel Purity Index (PPI) was used to find the most spectrally pure pixels. The PPI is computed by repeatedly projecting $n-D$ scatter plots on a random unit vector. The results of the PPI are usually used as input into the n-D Visualizer. Then, PPI values were computed from the MNF output by using the ENVI software. Higher PPI values represent pixels with higher purity and spectral extremity. The most spectrally pure pixels typically correspond to mixed endmembers. Separating purer pixels from more mixed pixels benefited separation and identification of endmembers (Wang, 2013).
After we have investigated the effects of PPI threshold on the final Cannabis detection, we used only the pixels with PPI values not less than 2000. This process has generated 32 pure pixels that were used as inputs in the n-Dimensional Visualizer in ENVI for the clustering process, which clustered the 32 pixels into distinct groups each one represents a kind of vegetation. Cannabis was recognized in the study area based on the ground-truth maps of Cannabis distribution in Morocco in 2003.

\subsection{Cannabis Detection}

Spectral Angle Mapper (SAM) was used for Cannabis detection in this study. SAM is defined as "a physically-based spectral classification that uses an n-D angle to match pixels to reference spectra. The algorithm determines the spectral similarity between two spectra by calculating the angle between the spectra and treating them as vectors in a space dimensionality equal to the number of bands". Since this method uses only the direction of a vector and not its length, it is insensitive to illumination and albedo effects (Exelis, 2014).

SAM was applied to the tested area data of Hyperion based on extracted endmember spectra in (section 3.3). In the beginning, we used the default angle threshold option of SAM in ENVI software which is $(0.100)$. This parameter defines the maximum acceptable angle between the endmember spectrum vector and the pixel vector. Hence, it will not classify pixels with an angle larger than the defined value in this threshold. In the SAM Alunite rule image, the pixel values of the rule image represent the spectral angle in radians from the reference spectrum for every class, which the lower spectral angles perform better matches to the endmember spectra (Kale, 2016).

\section{RESULTS AND DISCUSSIONS}

By using a PPI threshold of 2000, more endmembers were selected for the components of Cannabis and other forms of vegetation. From results that are representing in Figure 4, we can see a six-dimensional visualization of the spectra representative of Cannabis using the first $6 \mathrm{MNF}$ bands with high eigenvalues. Cannabis fields, as shown in red color, were identified by using the Spectral Angle Mapper (SAM), Using the default threshold option for the Single-Value and of 0.03 for maximum angle (radians). This maximum angle value was determined after a long process of trial and error to get the best classification results of Hyperion data in the Moroccan tested area.

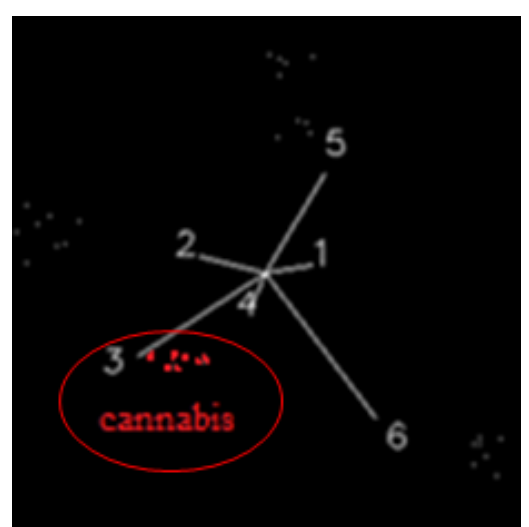

Figure 4. Pure Cannabis pixels' detection 
Among the 32 pure pixels that have been extracted from the study area; ten pixels were representing pure Cannabis spectra (as represented in figure 5Spectratra of extracted pixels were examined for similarity, then five Cannabis spectra, were finalized to detect Cannabis in the study area.

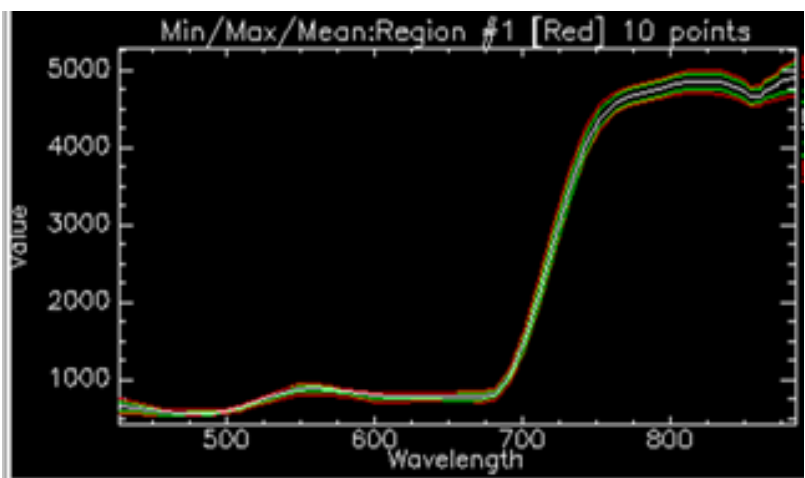

Figure 5. Cannabis Spectral profile of extracted endmembers

Cannabis detection results were represented in figures 6 for Hyperion data of 2003, figure 7 of 2009 Hyperion data in Morocco for positive Cannabis detection and figure 8 represented the Hyperion data of Algeria in 2004.

Positive Cannabis results were obtained from Moroccan sites represented in red in figures 6 and 7 . Whereas, no Cannabis detection was revealed from Algerian data in figure 8.

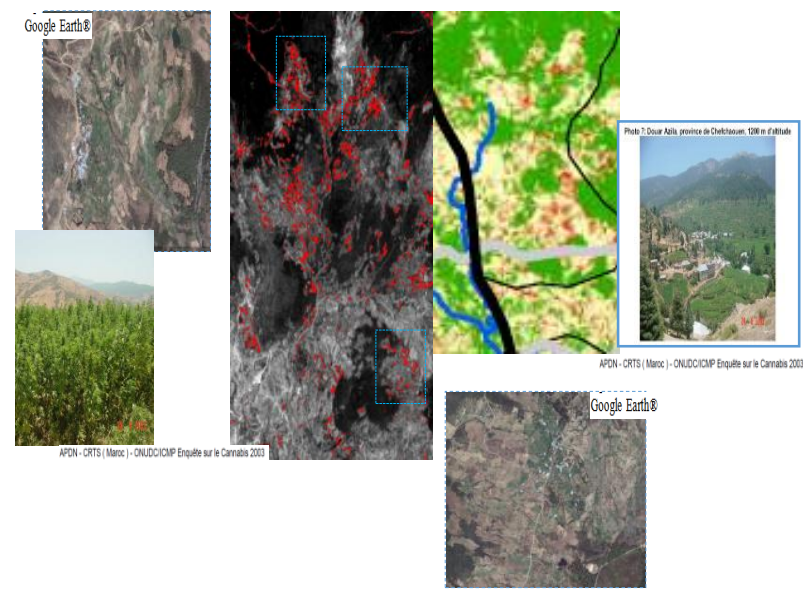

Figure 6. Cannabis detection in Morocco Hyperion data in 2003 compared to the ONUDC/ICMP Morocco Cannabis Survey report of the same year (UNODC, 2003), and Google Earth ${ }^{\circledR}$ high spatial resolution images (GoogleEarthPro, 2017a; GoogleEarthPro, 2017b)

The detection accuracy is assessed by comparing the results, with the map of Cannabis fields provided by UNODC, which conducted a detailed study on the cultivation of Cannabis in Morocco. This result demonstrates clearly that the Cannabis field distribution retrieved in this study looked quite similar to the interpretation of Moroccan Cannabis survey 2003 (UNODC, 2003), where the retrieved Cannabis field distribution was compared against a Cannabis field distribution map of this report.

Additionally, we used Google Earth ${ }^{\circledR}$ high spatial resolution images (GoogleEarth Pro., 2017a), to examine our results based on Cannabis plant shape and farming style in geo-tagged pictures. Our results were positive and matched with Google Earth ${ }^{\circledR}$ high spatial resolution images.

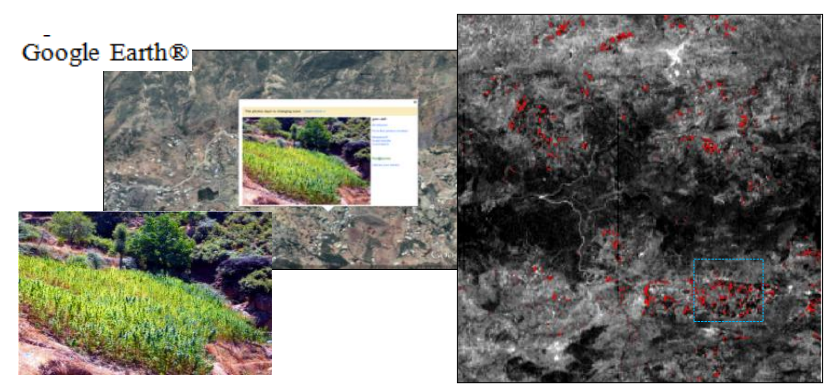

Figure 7. Cannabis data detection in Moroccan Hyperion data in 2009 compared to Google Earth ${ }^{\circledR}$ image published in the same year (GoogleEarthPro, 2017a; GoogleEarthPro, 2017b)

Finally, we applied SAM with our threshold on Hyperion images in well-known areas with negative Cannabis plantation in Algeria to avoid any false Cannabis detection using these spectra. Results for these tests were completely negative which indicates the applicability of these spectra to detect only Cannabis plantation in inaccessible areas.

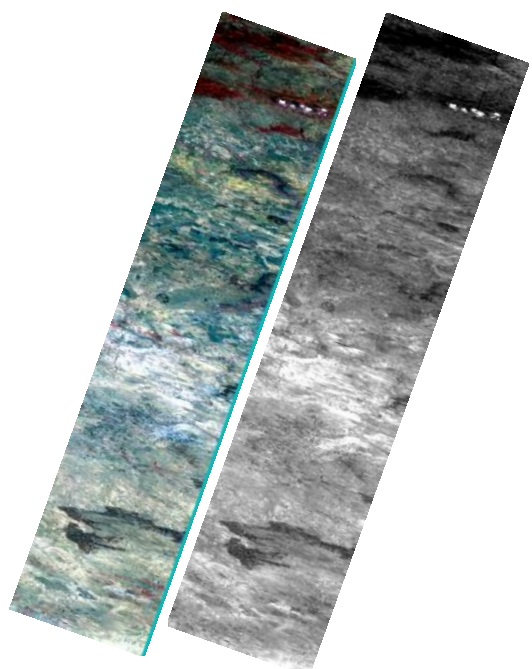

Figure 8. Cannabis negative detection in Algerian Hyperion data of 2004.

\section{CONCLUSION}

EO-1 Hyperion hyperspectral imagery was used in this study to collect Cannabis endmembers from well-known plantation fields in Morocco to obtain different spectra to be used in Cannabis plantation detection in inaccessible areas. After preprocessing Hyperion data, this study indicates that this hyperspectral imagery is capable of distinguishing Cannabis plants from several surrounding vegetation types. We extracted five different Cannabis spectra can detect Cannabis in VNIR. By using Spectral Angle Mapper (SAM). The results obtained the majority of agricultural (Cannabis) fields could be classified successfully by applying a Spectral Angle Mapper algorithm with 0.03 angle threshold. Therefore, the spectral behavior of various land cover types is very similar. However, the small space sample of many fields in the area produces numerous mixed pixels which further lower the pixel-based classification accuracy for the spatial resolution of $30 \mathrm{~m}$ in Hyperion. As a shortcoming of this research, we believe that SAM technique as a pixel-based classification does not represent the whole area of Cannabis fields due to the moderate spatial resolution of Hyperion data. Hence, we are planning to apply one or more 
sub-pixel based classification in our future work to improve illegal Cannabis plantation detection.

\section{ACKNOWLEDGMENT}

The work presented here was supported by the Natural Science Foundation of China (Grant No.61331016).

\section{REFERENCES}

Azaria, I., Goldschleger, N., and Ben-Dor, E., 2012. Identification of Cannabis plantations using hyperspectral technology. Israel Journal of Plant Sciences, 60(1-2), 77-83.

Beck, R., 2003. EO-1 User Guide v.2.3. http://eo1.usgs.gov/documents/EO1userguidev2pt320030715U C.pdf. Access date: 13.10.2017.

Boardman, J. W., and Kruse, F.A., 1994. Automated spectral analysis: A geologic example using AVIRIS data, north Grapevine Mountains, Nevada. Proceedings, Tenth Thematic Conference on Geologic Remote Sensing, Environmental Research Institute of Michigan, Ann Arbor, MI, p. I-407 - I418.

CBRC, 2007. Satellite Detection of Cannabis sativa Outdoor Grow Operations, Canadian Police Research Centre.

Chen, H., Han, T., Murdoch, M., and West C., 2003. Processing Hyperion and ALI for forest classification, IEEE Transactions on Geoscience and Remote Sensing, Vol. 41, pp. 1321-1331.

ENVI Development Team, 2015. Exelis Visual Information Solutions, Boulder, Colorado.

Exelis Visual Information Solutions, 2014. ENVI Classic Tutorial: Spectral Angle Mapper (SAM) and Spectral Information Divergence (SID) Classification, Boulder, Colorado: Exelis Visual Information Solutions.

Goodenough, D. G., Dyk, A., Niemann, K. O., Pearlman, J. S., Chen, H., Han, T., Murdoch, M., and West, C., 2003. Processing Hyperion and ALI for forest classification. IEEE transactions on geoscience and remote sensing, 41(6), 13211331.

GoogleEarthPro, 2017a.

http://www.google.com/earth/download/ge/

GoogleEarthPro 7.1.1.1871, 2017b. Morocco, Chefchaouen, $35^{\circ} 05^{\prime}$ 42.52"S, $5^{\circ} 27$ '05.69'W, Eye alt $13.67 \mathrm{Km}$. Digital Globe 2012. http://www.earth.google.com [June 26, 2012]. Data access: 20.12.2017.

Jupp, D.L.B., Datt, B., McVicar, T.R., Van Niel, T.G., Pearlman, J.S., Lovell, J.L. and King, E.A., 2003. Improving the analysis of Hyperion red-edge index from an agricultural area. Proceedings of SPIE, Vol. 4898, pp. 1-15.

Kale, K. V., 2016. Hyperspectral Data Processing and Analysis using ENVI/Python, Doctoral dissertation, Department of Computer Science and IT, Marathwada University, Aurangabad.
Noujdina, N.V., and Ustin, S.L., 2008. Mapping Downy Brome (Bromus tectorum) using multidate AVIRIS data. Weed Science, Vol. 56, pp. 173-179.

Somers, B., Asner, G.P., Laurent, T., and Coppin, P., 2011. Endmember variability in Spectral Mixture Analysis: A review. Remote Sensing of Environment, Vol. 115, No. 7, pp. 16031616.

Tsai, F., Lin E.K., and Yoshino, K., 2007. Spectrally segmented Principal Component Analysis of hyperspectral imagery for mapping invasive plant species. International Journal of Remote Sensing, Vol. 28, pp. 1023-1039.

Wang, J. J., 2013. Unsupervised Detection of Opium Poppy Fields in Afghanistan from EO-1 Hyperion Data. Master diss., University of New Brunswick.

UNODC, 2010. Afghanistan Cannabis survey 2009, United Nations Office on Drugs and Crime.

UNODC, 2003. Moroccan Cannabis survey 2003, United Nations Office on Drugs and Crime (In French)

USGS, 2017. https://earthexplorer.usgs.gov. Access date: 27.09.2017. 